\title{
Conocimientos previos acerca de métodos anticonceptivos y su relación con conocimientos adquiridos después de una intervención educativa con simulador ${ }^{1}$
}

\section{Institución: Universidad del Área Andina}

\author{
María Neyfeth Posada Morales ${ }^{2}$ \\ Bárbara de las Mercedes Mora Espinoza ${ }^{3}$
}

\section{COMO CITAR}

Posada, M. y Mora, B. (2015). Conocimientos previos acerca de métodos anticonceptivos y su relación con conocimientos adquiridos después de una intervención educativa con simulador. Rev. Enfermería Actual en Costa Rica, 28, 1-13. DOI: http://dx.doi.org/10.15517/revenf.v0i28.17203

\section{RESUMEN}

Introducción. El objetivo de este trabajo es relacionar conocimientos previos (pre-saberes) acerca de métodos anticonceptivos con los conocimientos adquiridos (saberes), después una intervención educativa con simulador. Método. Es un estudio realizado con 295 adolescentes escolarizados que cursan noveno, décimo y undécimo en una institución educativa pública. Es de tipo descriptivo. El proceso estuvo compuesto por tres fases; en la primera se midió conocimientos o saberes previos acerca de métodos anticonceptivos, mediante un cuestionario auto-informado; en la segunda se definió los contenidos y actividades de intervención para mejorar los conocimientos previos, y en la tercera fase de post-intervención educativa, se reevaluó los saberes adquiridos con el simulador.

Resultados. Se detectó que los pre-saberes en métodos anticonceptivos son defícientes independientemente de la edad y la escolaridad, lo cual requiere planeación, desarrollo y evaluación de la estrategia de aprendizaje colectivo y cooperativo mediante talleres. En cuanto a los post-saberes, se obtuvo una calificación de deficientes a regulares, en el caso del condón masculino, mientras que el reconocimiento de métodos modernos como parche, anillo vaginal, dispositivo intrauterino, entre otros, se dio gracias al contacto directo con el simulador.

Conclusiones. Se recalca que a pesar de las diferencias de edad y escolaridad, no hubo hallazgos relevantes que demostraran diferencias de conocimiento. En los pre-saberes, se encontró niveles de conocimiento deficientes sobre métodos anticonceptivos. El uso de simuladores como herramienta de aprendizaje demostró eficacia en los talleres; en cuanto a los simuladores posibilitan la ruptura de la barrera cognitiva, dado que el adolescente, en este caso, puede explorar y adquirir conocimiento al interaccionar con el objeto de aprendizaje.

Palabras clave: conocimientos-previos; estrategia-educativa; métodos-anticonceptivos; pre-saberes; post-saberes; simulador.

\footnotetext{
${ }^{1}$ Fecha de recepción: 13 de octubre del 2014

Fecha de aceptación: 15 de noviembre del 2014

${ }^{2}$ Enfermera. Fundación Universitaria del Área Andina, Seccional Pereira, Risaralda, Colombia. Correo electrónico: mariaposada@funandi.edu.co

${ }^{3}$ Enfermera. Fundación Universitaria del Área Andina, Seccional Pereira, Risaralda, Colombia. Correo electrónico: bmora@funandi.edu.co
} 


\title{
Previous knowledge about contraceptive methods and its relation with knowledge acquired after an educational intervention with simulator ${ }^{1}$
}

\author{
María Neyfeth Posada Morales ${ }^{2}$ \\ Bárbara de las Mercedes Mora Espinoza ${ }^{3}$
}

\section{Institution: Andean Area, University Foundation}

\section{CITED:}

Posada, M. y Mora, B (2015).Previus knowledge about contraceptive methods and its relation with knowledge acquired after an educational intervention with simulator. Rev. Enfermería Actual en Costa Rica, 28, 1-13. DOI:http://dx.doi.org/10.15517/revenf.v0i28.17203

\begin{abstract}
Introduction: The objective of this work is to relate previous knowledge about contraceptive methods with the acquired knowledge after an educational intervention with Simulator.

Method: It is a study of 295 school adolescents enrolled in ninth, tenth, and eleventh in a public educational institution. It is descriptive. The process consisted of three phases; the first was measured knowledge or previous knowledge about contraceptive methods, using a self-reported questionnaire; the second was defined the contents and activities of intervention for improving the background, and in the third phase of post-intervention educational re-evaluated the knowledge acquired with the simulator.

Result. Detected that the pre-knowledge in methods of contraception are poor regardless of age and schooling, which requires planning, development and evaluation of the strategy of collective and cooperative learning through workshops. As for the post-knowledge, was obtained a rating of poor to regular, in the case of the male condom, while the recognition of modern methods such as patch, vaginal ring, IUD, among others, by their physical appearance and location by direct contact with the simulator.

Conclusion. Stressing that despite differences of age and education, there were no relevant findings that demonstrated differences in knowledge. In the pre-knowledge, found levels of knowledge deficient about contraception. The use of simulators as a learning tool demonstrated effectiveness in the workshops; regarding simulators make possible rupture of the cognitive barrier, since the adolescent, in this case, can explore and acquire knowledge to interact with the aim of learning.
\end{abstract}

Keywords: previus-knowledge; pre-knowledge; contraceptive-methods; simulator; educational-strategy; post-knowledge.

\footnotetext{
${ }^{1}$ Date of receipt: October 13, 2014

Date of acceptance: November 15, 2014

${ }^{2}$ Nurse. Andean Area, University Foundation, Pereira Seccional. Risaralda, Colombia. E-mail: mariaposada@, funandi.edu.co

${ }^{3}$ Nurse. Andean Area, University Foundation, Pereira Seccional. Risaralda, Colombia. E-mail: bmora@,funandi.edu.co
} 


\section{.}

\section{INTRODUCCIÓN}

La adolescencia, periodo que transcurre entre "los 10 y 19 años de edad", según la OMS mencionado por UNFPA-Colombia (2008, p.23) es un fenómeno de muchos cambios y profundas transformaciones biológicas, psicológicas, sociales y cognitivas a los que se enfrentan los adolescentes, buscando fundamentalmente la identidad; si en esta tarea se fracasa, la persona será más susceptible de asumir comportamientos sexuales riesgosos, como el embarazo, el aborto inducido, las enfermedades e infecciones de transmisión sexual, morbilidad, discapacidad y mortalidad materna e infantil, asociada con el inicio de la práctica sexual a temprana edad y el desconocimiento de los métodos para prevenir el embarazo (Mancilla, 2012), aspectos que demuestran la necesidad que tienen los adolescentes de recibir información acerca de temas relacionados con la sexualidad, lo cual implica encontrar formas novedosas para abordar las necesidades de los adolescentes, siempre partiendo del saber previo (Higuera y Domínguez, 2007), para que el aprendizaje sea significativo, lo cual se convierte en una oportunidad para enfermería.

Dado lo anterior, como objetivo de esta investigación se pretende relacionar los conocimientos previos (pre-saberes) acerca de métodos anticonceptivos con los conocimientos adquiridos (saberes), después una intervención educativa con simulador.

\section{MATERIALES Y MÉTODO}

Se realizó en el año 2013 una investigación en una institución educativa pública del Departamento de Risaralda, municipio de Dosquebradas, con adolescentes institucionalizados; la entidad cuenta con una matrícula activa que corresponde a una población de 1370 que, según directoras, requerían formarse en cuanto a la responsabilidad sexual en los grados noveno, décimo y undécimo, con muestra no probabilística o por conveniencia (295 estudiantes). Es un estudio descriptivo.

El proceso estuvo compuesto por tres fases: en la primera, se midió el nivel de conocimiento básico a través de la aplicación de un cuestionario auto-informado que incluyó variables sociodemográficas y teóricoconceptuales sobre métodos anticonceptivos, previamente validado por tres expertos y experimentado en prueba piloto, de la cual se definieron los contenidos y actividades de intervención para mejorar los conocimientos (segunda fase). En esta segunda fase, se determinó la necesidad de enseñar los métodos anticonceptivos en varias sesiones, utilizando simuladores de "tarea básica" (task trainer simulator Laerdal y Limbs and Things), con los que los adolescentes podrían desarrollar cierta habilidad y destreza en el uso de los métodos anticonceptivos, actividad que se complementó con información acerca de mecanismos de acción, ventajas, desventajas, prevención de enfermedades e infecciones de transmisión sexual, contraindicaciones, entre otros.

Los simuladores fueron trasladados desde el Centro de simulación integral en salud de la Fundación Universitaria del Área Andina en Pereira, hasta la institución educativa municipal de Dosquebradas, Risaralda. Cada simulador sirvió para la muestra y la práctica de lo enseñado a los estudiantes, teniendo en cuenta que para ambas actividades se formaron subgrupos de adolescentes. 


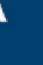

r

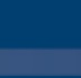

\section{Revista Electrónica Enfermería Actual de costa Rica}

Para desarrollar la tercera fase del proceso o post-intervención educativa en la cual se re-evaluó los saberes adquiridos por medio de los simuladores y desarrollo de contenidos, y considerando los estándares internacionales de simulación clínica avalados por la International Nursing Association for clinical simulation learning (INACSL), se diseñó una lista de chequeo del proceso por evaluar para establecer la apropiación de los diferentes métodos anticonceptivos, esta lista se aplicó a cada estudiante; posteriormente se llevó a cabo una retroalimentación, la cual se socializó de manera grupal.

\section{Consideraciones éticas}

La investigación mantuvo los parámetros éticos para estudios con seres humanos, incluyendo consentimiento informado, participación voluntaria, manejo confidencial de la información, empleo de evidencia disponible para la intervención educativa, y autorización de las instancias pertinentes; de igual forma, contó con el aval del comité de ética de la universidad.

\section{RESULTADOS}

Participaron 295 adolescentes escolarizados, de los cuales el 36.6\% se encontraba cursando noveno grado; el 33.2\%, décimo grado y el 30.2\%, undécimo grado. La edad de los participantes se definió según los parámetros de la OMS/OPS (2008), en adolescencia inicial (10 a 13 años), media (14 a 16 años) y tardía (17 a 20 años), etapas representadas en los grupos del estudio y que, a su vez, son relevantes porque las diferencias cognitivas del adolescente influyen en su salud sexual y reproductiva y en la forma en que asimile la información. (Véase tabla 1)

\section{Tabla 1}

Distribución de adolescentes según edad y escolaridad, 2013.

(Frecuencias absolutas y relativas)

\begin{tabular}{c|c|c|c|c|c|c|c}
\hline \multirow{2}{*}{ Edad } & \multicolumn{6}{|c|}{ Escolaridad } & \multirow{2}{*}{ Total } \\
\cline { 2 - 7 } & \multicolumn{2}{|c|}{ Noveno } & \multicolumn{2}{|c|}{ Décimo } & \multicolumn{2}{c}{ Undécimo } & $f_{i}$ \\
\cline { 2 - 7 } & $f_{i}$ & $h_{i}$ & $f_{i}$ & $h_{i}$ & $f_{i}$ & $h_{i}$ & 11 \\
\hline 10 a 13 años & 11 & 10,2 & - & - & - & - & 11 \\
\hline 14 a 16 años & 95 & 88,0 & 86 & 87,8 & 70 & 78,6 & 251 \\
\hline 17 a 20 años & 2 & 1,8 & 12 & 12,2 & 19 & 21,4 & 33 \\
\hline Total & $\mathbf{1 0 8}$ & $\mathbf{1 0 0 , 0}$ & $\mathbf{9 8}$ & $\mathbf{1 0 0 , 0}$ & $\mathbf{8 9}$ & $\mathbf{1 0 0 , 0}$ & $\mathbf{2 9 5}$ \\
\hline
\end{tabular}

Fuente: Base de datos

La distribución por género en cada uno de los grados fue equitativa, puesto que en general es política de la institución educativa mantener una relación 50:50 entre hombres y mujeres o con márgenes de diferencia estrecha. Lo anterior permitió establecer que la información obtenida tanto en los pre saberes como en los saberes, y la participación en la estrategia educativa, fueron abordadas desde la perspectiva de género y equidad, aspecto proclamado en la "Convención sobre la eliminación de todas las formas de discriminación contra la mujer (CEDAW 1979)", que se refiere a la educación en igualdad de condiciones, de modo que se elimine cualquier concepto estereotipado de los papeles masculino o femenino en todos los niveles de enseñanza (Artículo 10), y un 
derecho a la salud que garantice servicios de atención y orientación acerca de métodos anticonceptivos(Artículo 12).

Respecto de lo anterior, el Programa de Acción acordado en la "Conferencia internacional sobre la población y desarrollo" celebrada en El Cairo (CIPD 1994) en sus Capítulos VI y VII, expresa la necesidad de que tanto hombres como mujeres dispongan de información y de los medios para alcanzar un nivel más elevado concerniente a la salud y los derechos reproductivos para el ejercicio de la paternidad responsable, rubro que prioriza las necesidades de los adolescentes en este aspecto, dada su particularidad, vulnerabilidad por desinformación, conocimientos insuficientes o inadecuados y su tendencia a comportamientos sexuales riesgosos, carencias que motivan el mejoramiento de las estrategias educativas en salud sexual y reproductiva (Capítulo XII).

\section{PRE-SABERES}

En la tabla 2 se observa que los pre-saberes de los adolescentes en relación con los métodos anticonceptivos son deficientes en todos los grupos de escolaridad y de edad (temprana, media o tardía), deficiencia seguida de la calificación regular en lo que respecta a pre-saberes, resultado preocupante dado que el grupo de adolescentes es uno de los más vulnerables y expuestos a embarazos no deseados, no planificados o imprevistos, con riesgo de enfermedades de transmisión sexual, cuyo inicio de la práctica sexual es muy temprano (en general a los 16 años de edad), aun cuando no conocen de métodos anticonceptivos o cuando información o asesoramiento que manejan es deficiente.

\section{Tabla 2}

\section{Distribución de pre-saberes en métodos anticonceptivos según edad (OMS) y escolaridad, 2013}

\begin{tabular}{|c|c|c|c|c|c|c|c|c|c|c|c|c|c|c|c|c|c|c|c|c|c|}
\hline \multirow[b]{4}{*}{ Edad } & \multicolumn{20}{|c|}{ Escolaridad } & \multirow[b]{4}{*}{ Total } \\
\hline & \multicolumn{6}{|c|}{ Noveno } & \multicolumn{7}{|c|}{ Décimo } & & \multicolumn{6}{|c|}{ Undécimo } & \\
\hline & \multicolumn{2}{|c|}{ B } & \multicolumn{2}{|c|}{$\mathbf{R}$} & \multicolumn{2}{|c|}{ D } & \multirow[b]{2}{*}{ Total } & \multicolumn{2}{|c|}{ B } & \multicolumn{2}{|c|}{$\mathbf{R}$} & \multicolumn{2}{|c|}{ D } & \multirow[b]{2}{*}{ Total } & \multicolumn{2}{|c|}{ B } & \multicolumn{2}{|c|}{$\mathbf{R}$} & \multicolumn{2}{|c|}{ D } & \\
\hline & $f_{i}$ & $h_{\mathrm{i}}$ & $f_{i}$ & $h_{\mathrm{i}}$ & $f_{i}$ & $h_{\mathrm{i}}$ & & $f_{i}$ & $h_{\mathrm{i}}$ & $f_{\mathrm{i}}$ & $h_{\mathrm{i}}$ & $f_{i}$ & $h_{\mathrm{i}}$ & & $f_{i}$ & $h_{\mathrm{i}}$ & $f_{i}$ & $h_{\mathrm{i}}$ & $f_{i}$ & $h_{\mathrm{i}}$ & \\
\hline $\begin{array}{c}10 \text { a } 13 \\
\text { años }\end{array}$ & 2 & 18,2 & 3 & 27,3 & 6 & 54,5 & 11 & - & - & - & - & - & - & - & - & - & - & - & - & - & - \\
\hline $\begin{array}{c}14 \text { a } 16 \\
\text { años }\end{array}$ & 16 & 16,8 & 9 & 9,5 & 70 & 73,7 & 95 & 9 & 10,5 & 30 & 34,9 & 47 & 54,6 & 86 & 8 & 11,4 & 21 & 30 & 41 & 58,6 & 70 \\
\hline Total & 20 & 18,5 & 12 & 11,1 & 76 & 70,4 & 108 & 11 & 11,2 & 33 & 33,7 & 54 & 55,1 & 98 & 12 & 13,5 & 26 & 29,2 & 51 & 57,3 & 89 \\
\hline
\end{tabular}

Fuente: Base de datos. Clasificación de los pre-saberes: B (Buenos), R (Regulares) y D (Deficientes)

\section{Análisis de la intervención desde la estrategia educativa con simulación.}

En el análisis de pre-saberes acerca de métodos anticonceptivos, se confirmó que no existen diferencias al respecto entre los diferentes grupos de escolaridad; los saberes que comparten en un gran porcentaje son 


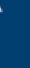

deficientes, seguidos de conocimientos regulares, lo cual no fue significativo para establecer intervenciones educativas individualizadas de grupo, sino estrategias de aprendizaje colectivo y cooperativo, vividas durante los talleres.

La estrategia educativa desarrollada se basó en la Teoría del Aprendizaje Significativo (Amaya, 2010, p.208), y consideró:

- Los pre-saberes o conocimientos previos en torno al tema: se clasificó en buenos, regulares y deficientes, para reconocer los preconceptos de los estudiantes frente al tema.

- Material significativo: Ayuda al estudiante a anclar los conocimientos nuevos con los previos, porque si no sucede, no se transformará el conocimiento. Además, se emplea los simuladores como una herramienta de aprendizaje que contribuye a descubrir y reconstruir información y a asimilar de manera vivencial la experiencia educativa.

- Tiempo: significa que se requiere un proceso de desarrollo con el objeto de conocimiento que dependerá del estilo de aprendizaje del estudiante.

- Facilitación: necesaria para que el estudiante acceda no solo a la información teórica, sino que interacciona con el objeto de aprendizaje para construir su propio saber.

- Retroalimentación: es indispensable brindar un acompañamiento dirigido cuando el estudiante no ha logrado la competencia esperada y es necesario presentarle el contenido en otra didáctica que le ilustre y demuestre el saber no alcanzado.

- Interacción: se constituye en la base esencial y clave del aprendizaje significativo: no se concibe un aprendizaje significativo sin la interacción docente-estudiante, porque es el docente quien orienta, direcciona y explica cómo lograr el aprendizaje con la estrategia adecuada.

Desde esta perspectiva, cada uno de dichos componentes fue vital en la planeación, desarrollo y evaluación formativa de la estrategia de enseñanza de los métodos anticonceptivos.

En la tabla 3 se evidencia, que independientemente del grado de escolaridad, los conocimientos previos o pre-saberes sobre cada uno de los métodos anticonceptivos son deficientes. Los métodos anticonceptivos más desconocidos son los modernos como parche, anillo vaginal, implante sub-dérmico y dispositivos intrauterinos hormonales o de cobre; al respecto, llama la atención que los conocimientos previos sobre el método más comercializado como es el condón masculino, se encuentren entre regular y deficientes, lo cual es alarmante si se considera lo descrito por la literatura en cuanto a la edad de inicio de relaciones sexuales en adolescentes: entre los 13 y 18 años de edad (Loza y Gómez, 2008).

Por otra parte, de los pre-saberes clasificados como "buenos" en todos los métodos anticonceptivos no superan en general el 40\%, por lo tanto, el adolescente se está enfrentando o se va a enfrentar a la práctica sexual con el mínimo conocimiento de los métodos anticonceptivos, en caso de que decida utilizarlos, de modo que se expone a una práctica insegura y de riesgo. 


\section{Revista Electrónica Enfermeria Actual de costa Rica}

\section{Tabla 3}

Distribución de pre-saberes por método anticonceptivo y escolaridad, 2013.

(Frecuencias absolutas y relativas)

\begin{tabular}{|c|c|c|c|c|c|c|c|c|c|c|c|c|c|c|c|c|c|c|c|}
\hline \multirow{4}{*}{$\begin{array}{c}\text { Pre-saber } \\
\text { Método } \\
\text { anticonceptivo }\end{array}$} & \multicolumn{18}{|c|}{ Escolaridad } & \\
\hline & \multicolumn{6}{|c|}{ Noveno } & \multicolumn{6}{|c|}{ Décimo } & \multicolumn{6}{|c|}{ Undécimo } & \\
\hline & \multicolumn{2}{|c|}{ B } & \multicolumn{2}{|c|}{$\mathbf{R}$} & \multicolumn{2}{|c|}{ D } & \multicolumn{2}{|c|}{ B } & \multicolumn{2}{|c|}{$\mathbf{R}$} & \multicolumn{2}{|c|}{ D } & \multicolumn{2}{|c|}{ B } & \multicolumn{2}{|c|}{$\mathbf{R}$} & \multicolumn{2}{|c|}{ D } & \\
\hline & $f_{i}$ & $h_{i}$ & $f_{i}$ & $h_{i}$ & $f_{i}$ & $h_{i}$ & $f_{i}$ & $h_{i}$ & $f_{i}$ & $h_{i}$ & $f_{i}$ & $h_{i}$ & $f_{i}$ & $h_{i}$ & $f_{i}$ & $h_{i}$ & $f_{i}$ & $h_{i}$ & \\
\hline Píldora de emergencia & - & - & 9 & 8,3 & 99 & 91,7 & 12 & 12,2 & 29 & 29,6 & 57 & 58,2 & 6 & 6,8 & 33 & 37,0 & 50 & 56,2 & \\
\hline Pastas anticonceptivas & - & - & 31 & 28,7 & 77 & 71,3 & 26 & 26,6 & 11 & 11,2 & 61 & 62,2 & 11 & 12,3 & 38 & 42,7 & 40 & 45,0 & \\
\hline Inyección anticonceptiva & - & - & 42 & 38,9 & 66 & 61,1 & 19 & 19,4 & 17 & 17,3 & 62 & 63,3 & 16 & 29,0 & 29 & 32,6 & 44 & 49,4 & \\
\hline Parche anticonceptivo & - & - & 4 & 3,7 & 104 & 96,3 & 2 & 2,0 & - & - & 96 & 98,0 & 6 & 22,0 & 22 & 24,7 & 61 & 68,5 & \\
\hline Implante subcutáneo & - & - & 14 & 13,0 & 94 & 87,0 & 6 & 6,2 & 11 & 11,2 & 81 & 82,6 & 9 & 25,0 & 25 & 28,0 & 55 & 61,8 & \\
\hline Anillo vaginal & - & - & 3 & 2,8 & 105 & 97,2 & 4 & 4,1 & 31 & 31,6 & 63 & 64,3 & 6 & 18,0 & 18 & 20,2 & 65 & 73,0 & \\
\hline Dispositivo intrauterino & - & - & 4 & 3,7 & 104 & 96,3 & - & - & 16 & 16,3 & 82 & 83,7 & 11 & 12,4 & 21 & 23,6 & 57 & 64,0 & \\
\hline Condón femenino & - & - & 42 & 39,0 & 29 & 26,8 & 31 & 31,6 & 37 & 37,8 & 30 & 30,6 & 30 & 33,7 & 39 & 43,8 & 20 & 22,5 & \\
\hline Condón masculino & - & - & 65 & 60,2 & 31 & 28,7 & 10 & 10,2 & 25 & 25,5 & 63 & 64,3 & 16 & 18,0 & 25 & 28,0 & 48 & 54,0 & \\
\hline Espermicidas & - & - & 17 & 15,7 & 91 & 84,4 & 3 & 3,1 & 26 & 26,5 & 69 & 70,4 & 9 & & 24 & 27,0 & 56 & 63,0 & \\
\hline Total & & & & 108 & & & & & & 8 & & & & & & 9 & & & \\
\hline
\end{tabular}

Fuente: Base de datos

\section{Análisis post-saberes.}

La tabla 4 muestra que, después de la estrategia educativa, los conocimientos sobre métodos anticonceptivos pasaron de deficientes a regulares, en todos los grupos participantes, por tanto, se confirma que la experiencia con simuladores les permite apropiar información, no obstante, la conducta del estudiante sigue siendo pasiva, es decir, receptiva con participación aclaratoria, mas no de profundización conceptual, transformación que sería ideal para observar cuánto conocimiento fue apropiado por el sujeto durante el proceso (Ruiz, Muller y Guevara, 2009).

Tabla 4

Distribución de saberes en métodos anticonceptivos según edad (OMS) y escolaridad, 2013.

(Frecuencias absolutas y relativas)

\begin{tabular}{|c|c|c|c|c|c|c|c|c|c|c|c|c|c|c|c|c|c|c|c|c|c|}
\hline \multirow{4}{*}{ Edad } & \multicolumn{21}{|c|}{ Escolaridad } \\
\hline & \multicolumn{6}{|c|}{ Noveno } & \multirow[b]{3}{*}{ Total } & \multicolumn{6}{|c|}{ Décimo } & \multirow[b]{3}{*}{ Total } & \multicolumn{6}{|c|}{ Undécimo } & \multirow[b]{3}{*}{ Total } \\
\hline & \multicolumn{2}{|c|}{ B } & \multicolumn{2}{|c|}{$\mathbf{R}$} & \multicolumn{2}{|c|}{ D } & & \multicolumn{2}{|c|}{ B } & \multicolumn{2}{|c|}{$\mathbf{R}$} & \multicolumn{2}{|c|}{ D } & & \multicolumn{2}{|c|}{ B } & \multicolumn{2}{|c|}{$\bar{R}$} & \multicolumn{2}{|c|}{ D } & \\
\hline & $f_{i}$ & $h_{i}$ & $f_{i}$ & $h_{i}$ & $f_{i}$ & $h_{i}$ & & $f_{i}$ & $h_{i}$ & $f_{i}$ & $h_{i}$ & $f_{i}$ & $h_{i}$ & & $f_{i}$ & $h_{i}$ & $f_{i}$ & $h_{i}$ & $f_{i}$ & $h_{i}$ & \\
\hline Io a I3 años & 3 & 27,3 & 6 & 4,5 & 2 & 18,2 & 11 & - & - & - & - & - & - & - & - & - & - & - & - & - & - \\
\hline I/4 a I6 años & 12 & 12,6 & 33 & 34,8 & 50 & 2,6 & 95 & 11 & 12,8 & 43 & 50,0 & 32 & 37,2 & 86 & 16 & 22,9 & 29 & 41,4 & 25 & 35,7 & 70 \\
\hline 17 a 20 años & 2 & 100,0 & - & 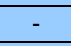 & - & 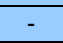 & 2 & 3 & 25,0 & \begin{tabular}{|l|}
7 \\
\end{tabular} & 58,3 & 2 & 16,7 & 12 & 5 & 26,3 & \begin{tabular}{|l|}
11 \\
\end{tabular} & 58,9 & 3 & \begin{tabular}{|l|}
15,8 \\
\end{tabular} & 19 \\
\hline Total & 17 & 15,8 & 39 & 36,1 & 52 & 48,1 & 108 & 14 & 14,3 & 50 & 51,0 & 34 & 34,7 & 98 & 21 & 26,3 & \begin{tabular}{|l|}
40 \\
\end{tabular} & 45,0 & 28 & 31,4 & 89 \\
\hline
\end{tabular}

Fuente: Base de datos. Clasificación de los presaberes: B (Buenos), R (Regulares) y D (Deficientes). 


\section{Revista Electrónica Enfermeria Actual de costa Rica}

La tabla 5 muestra que después de la intervención con la estrategia educativa con simuladores, hubo cambios en los saberes: el más significativo fue respecto del condón masculino, que pasó de deficiente a regular, aparte de que un porcentaje más bajo pasó a "bueno". En este caso, los adolescentes tuvieron la oportunidad de realizar prácticas sobre el simulador (pene), y manipular el condón desde su blíster hasta que fue desechado; ese contacto directo es el que permite un aprendizaje más significativo, no solamente por el contacto directo, sino porque se aprende del error, se disipa dudas y se establecen relaciones entre la realidad y la simulación.

Los métodos anticonceptivos modernos, que en los pre-saberes eran desconocidos y deficientes como el parche, anillo vaginal, implante sub-dérmico y dispositivos intrauterinos hormonales o de cobre, empezaron a ser reconocidos por algunos adolescentes, por su aspecto físico y ubicación, particularidades que fueron reforzadas con el uso de los simuladores, razón por la que es necesario continuar con esta estrategia en la enseñanza, incorporando nuevos conceptos y retroalimentado el aprendizaje.

Los saberes acerca de métodos anticonceptivos hormonales como las píldoras, inyecciones y píldoras de emergencia continúan siendo deficientes y regulares, aspecto que preocupa, debido a su uso indiscriminado, la automedicación, desconocimiento del uso, de los efectos adversos y de la necesidad de control médico.

\section{Tabla 5}

\section{Distribución de saberes post intervención educativa por método} anticonceptivo y escolaridad, 2013.

(Frecuencias absolutas y relativas)

\begin{tabular}{|c|c|c|c|c|c|c|c|c|c|c|c|c|c|c|c|c|c|c|c|}
\hline \multirow{4}{*}{$\begin{array}{c}\text { Post-saber } \\
\text { Método } \\
\text { anticonceptivo }\end{array}$} & \multicolumn{18}{|c|}{ Escolaridad } & \\
\hline & \multicolumn{6}{|c|}{ Noveno } & \multicolumn{6}{|c|}{ Décimo } & \multicolumn{6}{|c|}{ Undécimo } & \\
\hline & \multicolumn{2}{|c|}{ B } & \multicolumn{2}{|c|}{$\mathbf{R}$} & \multicolumn{2}{|c|}{ D } & \multicolumn{2}{|c|}{ B } & \multicolumn{2}{|c|}{$\mathbf{R}$} & \multicolumn{2}{|c|}{ D } & \multicolumn{2}{|c|}{ B } & \multicolumn{2}{|c|}{$\mathbf{R}$} & \multicolumn{2}{|c|}{ D } & \multirow[b]{4}{*}{ Г } \\
\hline & $f_{i}$ & $h_{i}$ & $\overline{f_{i}}$ & $h_{i}$ & $\overline{f_{i}}$ & $\overline{h_{i}}$ & $\overline{f_{i}}$ & $\overline{h_{i}}$ & $\overline{f_{i}}$ & $h_{i}$ & $\overline{f_{i}}$ & $\overline{h_{i}}$ & $\overline{f_{i}}$ & $\overline{h_{i}}$ & $\overline{f_{i}}$ & $h_{i}$ & $\overline{f_{i}}$ & $\overline{h_{i}}$ & \\
\hline Píldora de emergencia & 6 & 5,6 & 32 & 29,6 & 70 & 64,8 & 14 & 14,3 & 43 & 43,9 & 41 & 41,8 & 17 & 19,1 & 46 & 51,7 & 26 & 29,2 & \\
\hline Pastas anticonceptivas & 4 & 3,7 & 46 & 42,6 & 58 & 53,7 & 29 & 30,0 & 27 & 27,1 & 42 & 42,9 & 13 & 14,6 & 43 & 48,3 & 33 & 37,1 & \\
\hline Inyección anticonceptiva & 3 & 2,7 & 50 & 46,3 & 55 & 51,0 & 21 & 21,4 & 26 & 26,5 & 51 & 52,1 & 26 & 29,2 & 40 & 45,0 & 23 & 25,8 & D \\
\hline Parche anticonceptivo & 7 & 6,6 & 22 & 20,3 & 79 & 73,1 & 6 & 6,1 & 17 & 17,6 & 75 & 76,3 & 25 & 28,1 & 34 & 38,2 & 30 & 33,7 & $\mathbf{T}$ \\
\hline Implante subcutáneo & 4 & 3,8 & 20 & 18,5 & 84 & 77,7 & 12 & 12,2 & 21 & 21,5 & 65 & 66,3 & 10 & 11,2 & 36 & 40,4 & 43 & 48,3 & \\
\hline Anillo vaginal & 8 & 7,5 & 26 & 24,0 & 74 & 68,5 & 19 & 19,4 & 47 & 48,0 & 32 & 32,6 & 13 & 14,6 & 31 & 34,8 & 45 & 50,6 & L \\
\hline Dispositivo intrauterino & 4 & 3,8 & 20 & 18,5 & 84 & 77,7 & 8 & 8,2 & 32 & 32,7 & 58 & 59,1 & 19 & 21,3 & 43 & 48,3 & 27 & 30,4 & \\
\hline Condón femenino & 40 & 37,2 & 30 & 27,7 & 38 & 35,1 & 10 & 10,2 & 32 & 32,7 & 56 & 57,1 & 21 & 24,0 & 56 & 63,0 & 12 & 13,0 & \\
\hline Condón masculino & 21 & 19,5 & 58 & 53,7 & 29 & 26,8 & 40 & 40,8 & 47 & 48,0 & 11 & 11,2 & 37 & 41,6 & 49 & 55,0 & 3 & 3,4 & \\
\hline Espermicidas & 9 & 8,4 & 29 & 26,8 & 70 & 64,8 & 8 & 8,2 & 37 & 37,7 & 53 & 54,1 & 11 & 12,3 & 31 & 34,8 & 47 & 52,9 & \\
\hline Total & & & & 08 & & & & & & 8 & & & & & & 39 & & & 295 \\
\hline
\end{tabular}

Fuente: Base de datos

Considerando lo mencionado, es posible afirmar que la simulación se convierte en una necesidad para la enseñanza de los métodos anticonceptivos dirigida a los adolescentes, ya que los participantes no solo entran en contacto directo con el simulador, sino que pueden repetir el proceso, corregir y perfeccionar su uso y, a la vez, asociar beneficios, desventajas, modo de acción, entre otros aspectos. 


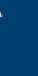

\section{DISCUSIÓN}

La falta de conocimientos en los temas mencionados se traduce en 8 a 30 millones de embarazos en el mundo por año, debido al fracaso en el uso de métodos anticonceptivos y su inexperiencia, datos que son confirmados por Gómez, Molina, y Zamberlin (2011) aspectos que contemplan, quienes afirman que en cuanto a los pre-saberes, la Teoría del Aprendizaje Significativo plantea que son el reflejo de lo que el adolescente ha oído, leído, observado, vivenciado o experimentado, por consiguiente, son fundamentales para poder construir un nuevo conocimiento sobre el ya existente; sin embargo, en relación con el tema de los métodos anticonceptivos no existe claridad teórico conceptual, tal como se muestra en la tabla 2, entonces el proceso de aprendizaje se ve afectado, al no poder establecer relaciones y asociaciones entre ambos, lo cual Aussubel, citado por Ballester (2002, p.16), denomina "aprendizaje significativo".

Respecto de los pre-saberes y de acuerdo con Demenjó (2006), es más provechoso construir un nuevo conocimiento de otro ya existente que incluya lo que ha leído, oído, observado o bien experimentado durante la vida, para que sea más significativo y se establezca relaciones entre saberes que, a su vez, permite a los educadores diseñar estrategias educativas basadas en las necesidades de la propia población, incluyendo conceptos más elaborados, que aumenten la posibilidad de que el adolescente relacione su saber previo con el saber nuevo hasta lograr un aprendizaje basado en la reflexión y en la aplicación en su propia vida. En este caso, para todos los grupos de edad se toma como punto común el que los participantes compartan saberes previos desconocidos, semejanza que implicó un aprendizaje más lento y la necesidad de encuentros más frecuentes en relación con el tema.

En el análisis de los resultados obtenidos respecto de los post-saberes, los investigadores Moccia y Medina (2006) encontraron en su estudio que el 97\% de los adolescentes conocían de la existencia de los métodos de planificación familiar, pero alrededor del $22 \%$ los usaban de forma incorrecta debido a que no habían sido educados acerca del tema; de lo anterior se concluye que las tareas educativas para adolescentes tienen éxito si son realizadas por personal preparado para dicho objetivo, tarea que debería retomar el personal de salud, el cual cuenta con un compromiso social y competencias en relación con la salud sexual y reproductiva.

En su estudio, Fétis, Bustos, Lanas, Baeza, Contreras, Hebel y Marucich (2008), establecieron que los adolescentes tienen conocimiento sobre métodos anticonceptivos, pero que la prevalencia del uso es muy baja (37\%), debido al desconocimiento y al poco apoyo de los profesionales de la salud en cuanto a la información, puesto que solo el $5.8 \%$ la recibe de parte de estos.

Con respecto a la edad, en el estudio de Cruz y Cabrera (2010), los resultados mostraron que el 64,4\% de los adolescentes creen que la edad más oportuna para aprender acerca de los métodos anticonceptivos es entre 12 -18 años, dado que consideran que las personas tienen criterios formados; luego, el 22,6\% cree que la edad indicada está entre los 7 y 12 años y el 12\%, que debe ser después de los 19 años. En tales edades, analizadas desde el punto de vista de Schutt y Maddaleno (2003), existen profundos cambios biológicos, psicológicos, sociales y cognitivos, que avanzan desde el pensamiento concreto en la adolescencia temprana o inicial hasta pensamientos lógicos y formales en la adolescencia tardía, etapa en la que hay más interés por temas de sexualidad, los cuales requieren acompañamiento, información, asesoría y orientación, desde etapas muy 
tempranas, porque la sexualidad se ha entendido como genitalidad y no como el proceso inherente y connatural al desarrollo del ser humano.

La educación sexual efectiva debe tener, por lo tanto, características especiales en las que se involucren los adolescentes y jóvenes, así como la familia, primera educadora, y la institución educativa, dado que ambas entidades comparten la responsabilidad que existe frente a la infancia y la adolescencia en su crecimiento y desarrollo, y contribuyen a desarrollar habilidades para la vida y desarrollo del proyecto de vida de los adolescentes, al punto de que esa faena se considera un compromiso de todos, como lo señala el CONPES 147 (2011) acerca de la prevención del embarazo y el logro de un proyecto de vida.

Según la última Encuesta Nacional de Demografía y Salud ENDS (Pro-familia 2010), se aplicó una encuesta a mujeres en edad fértil (13 a 49 años), con la que se pretendía medir el nivel de información sobre métodos anticonceptivos, de lo que se ubicó que no existían diferencias significativas, debido a que no se preguntaba sobre calidad de conocimiento, solo se leía el nombre del método y a continuación se le exponía una breve descripción y se le preguntaba si había oído hablar al respecto. Desde esta perspectiva, "conocer un método" no significa necesariamente que la mujer tenga un buen nivel de conocimiento, en el sentido de saber en forma adecuada qué es el método, cómo obra, cuáles son sus posibles contraindicaciones, cuáles sus probables efectos colaterales y qué se debe hacer o a dónde acudir en el caso que se lleguen a presentar; basta con que, por lo menos, lo conozca de nombre o tenga una idea vaga de cómo se usa; en otras palabras no es una medición de la calidad de conocimiento, de lo que se colige que, en efecto, hay información pero su calidad es ineficiente.

De acuerdo con la encuesta, hay claridad en términos de información pero no en apropiación de los métodos anticonceptivos, por tanto, se demuestra que no hay referentes nacionales de efectividad y eficacia en el conocimiento de los métodos como tal. Los datos aportados por Pro-familia son similares a los detectados en relación con los pre-saberes de los adolescentes objeto del estudio, quienes manejan información deficiente, por no contar con el objeto de conocimiento (métodos) en la prueba diagnóstica.

La estrategia educativa empleada en esta investigación demostró que aunque la simulación no reemplaza las situaciones reales, sí posibilita el aprendizaje o la adquisición de nuevos conocimientos significativos, obtenidos a través de un objeto virtual de aprendizaje (OVA), el cual cumple los elementos didácticos que no necesariamente deben estar mediados por una tecnología computarizada, sino por cualquier objeto que guarde similitud con la realidad, por ende, se constituye en un objeto real de aprendizaje, siempre y cuando cuente con el acompañamiento y la asesoría para el descubrimiento del saber que proyecta. Tal información fue corroborada con el uso del simulador, que demostró confiabilidad y seguridad respecto de la apropiación del conocimiento, al ofrecer la oportunidad de conocer e interaccionar con el objeto de estudio en forma continua y repetitiva hasta que cada sujeto construyó su propio conocimiento, remodela su información y, en caso necesario, usa la retroalimentación para que el estudiante alcance el nivel de conocimiento requerido acerca del tema.

En síntesis, el simulador, el cual incluye videos y modelos de entrenamiento, entre otros elementos, representa una herramienta segura y confiable en cualquier contexto educativo para cualquier tema, sobre todo si se trata de aquellos en los que el adolescente tiene miedo de preguntar pero, a la vez, la seguridad de que necesita tener claro un tema, como el de los anticonceptivos y otros inherentes a su sexualidad. Conocer más en relación con dicho tópico lo desinhibe, lo motiva a interaccionar y lo más importante es que con esta estrategia hace un 
aprendizaje colectivo y, mejor aún, contribuye a lograr el sexto Objetivo de Desarrollo del Milenio

(CONPES Social 140, 2012): "mejorar la salud sexual y reproductiva".

\section{CONCLUSIONES}

- En cuanto a las características sociodemográficas del grupo de estudio, existen diferencias de edad y género debido a los diferentes grados que formaron la muestra, mas no por ello se encontró hallazgos relevantes que demostraran diferencia de conocimiento con las dos variables mencionadas y que son inherentes a los temas de sexualidad.

- En los presaberes, el conocimiento acerca de los anticonceptivos que tienen los estudiantes objeto de este estudio, refleja serias carencias en torno al tema.

- La utilización de un simulador como herramienta didáctica en el aprendizaje significativa fue eficaz durante los talleres del tema de anticonceptivos, porque se pasó de contar con ciertos saberes previos, a asimilar los métodos en sí.

- En los resultados postsaberes, se considera significativa la apropiación de nuevos saberes, sin embargo, es necesario realizar nuevos abordajes pedagógicos que permitan llevar a un nivel de formación integral no solo de anticonceptivos, sino otros temas inherentes relacionados de manera directa.

- La enseñanza con simuladores posibilita la ruptura de la barrera cognitiva, ya que el estudiante explora y adquiere el conocimiento interaccionando con el objeto de aprendizaje, lo cual favorece su asimilación y la seguridad para cuestionar, confrontar y, sobre todo, afianzar su propio saber.

\section{REFERENCIAS BIBLIOGRAFICAS}

Amaya, A. (2010). Universidad Médica. Simulación clínica: Aproximación pedagógica. Bogotá, Colombia. Volumen 51 No. 2. Pág. 208. Recuperado de:

http://www.redalyc.org/pdf/2310/231016391008.pdf

Ballester, A. (2002). El aprendizaje significativo en la práctica: cómo hacer el aprendizaje significativo en el aula. Madrid. Recuperado de:

http://www.aprendizajesignificativo.es/mats/El aprendizaje significativo en la practica.pdf

CONPES Social 140. (2012) Modificación a CONPES Social 91 de 14 junio 2005 "Metas y Estrategias de Colombia para el logro de los Objetivos Desarrollo del Milenio. Bogotá 2012.

CONPES 147 (2011). Formulación de estrategia para la prevención del embarazo y proyecto de vida. Bogotá. Septiembre 2011.

Cruz, A. y Cabrera, D. (2010). Conocimientos sobre el uso de métodos anticonceptivo y su relación con riesgos reproductivos en adolescentes de los quintos y sextos cursos del Colegio Técnico "Federico Páez".Tesis para optar al título de Licenciada en Enfermería. Universidad del Norte. Facultad de Ciencias de la Salud. Escuela de Enfermería. Ibarra, Ecuador. Recuperado de: http://repositorio.utn.edu.ec/handle/123456789/662 


\section{Revista Electrónica Enfermeria Actual de costa Rica}

Demejó, M. (2006). El proceso cognitivo y el aprendizaje profesional. Revista Educación Médica. 8 (1), pp.11-16. Recuperado de:

http://scielo.isciii.es/pdf/edu/v9n1/colaboracionl3.pdf?iframe=true\&width $=90 \% \&$ height $=90 \%$

Encuesta Nacional de Demografía y Salud ENDS (2010). Profamilia. Capítulo 6. Planificación Familiar: Conocimiento y uso de métodos. Recuperado de:

http://www.profamilia.org.co/encuestas/Profamilia/Profamilia/images/stories/PDF-capitulos/Capitulo-6.pdf

Fétis, G., Bustos, L., Lanas, F., Baeza, B., Contreras, J., Hebel, E., Marucich, C.(2008). Factores asociados al uso de anticonceptivos en estudiantes de enseñanza media de la comuna de Temuco. Revista Chilena de Obstetricia y Ginecologia. 73(6), pp. 362-369. Recuperado de: http://www.scielo.cl/pdf/rchog/v73n6/art02.pdf

Gómez, I., Molina, R., Zamberlin, N. (2011). Factores relacionados con el embarazo y la maternidad en menores de 15 años en América Latina y el Caribe. Federación Latinoamericana de sociedades de obstetricia y ginecología (FLASOG). 2011. Lima, Perú. ISBN: 978-612-45897-68, pp. 17, 23, 24, 27,28. Recuperado de: http://www.unal.edu.co/bioetica/documentos/2011/Maternidad.pdf

Higuera, F., Domínguez, Z. (2007). Opinión que tienen las adolescentes a cerca de la atención en la consulta de planificación familiar en el Instituto Nacional de Puericultura. Tesis para optar al título de Licenciado en Enfermería. Universidad Central de Venezuela. Facultad de Medicina. Escuela de Enfermería. Caracas, Venezuela. Recuperado de:

http://saber.ucv.ve/xmlui/bitstream/123456789/453/1/Tesis\%20Completa\%20(Planificaci\%C3\%B3n\%20Familiar).pd f

Loza, S., Gómez, E. (2008). Barreras y estrategias para la investigación en salud sexual: una experiencia con adolescentes en escuelas públicas. Revista Mexicana de Investigación Educativa, 13(36), pp. 203-22. México. ISSN 1405-666. Recuperado de: http://www.redalyc.org/pdf/140/14003609.pdf

Mancilla, J. (2012). Embarazo en adolescentes. Vidas en riesgo. Revista de Perinatología y reproducción humana. México D.C. 26(1), pp.5- 7. Recuperado de: http://www.medigraphic.com/inper http://www.medigraphic.com/pdfs/inper/ip2012/ip121a.pdf

Moccia, A., Medina, R. (2006). ¿Qué saben los adolescentes a cerca de los métodos anticonceptivos y cómo los usan? Revista Médica del Uruguay. 22(3), pp.185-190. Recuperado de: http://www.rmu.org.uy/revista/2006v3/art4.pdf . http://www.rmu.org.uy/revista/22/3/2/es/4/resumen/

Naciones Unidas. Departamento de Asuntos Económicos y Sociales. Fondo de Población de las Naciones UNFPA. (1994). Informe de la Conferencia Internacional sobre la Población y Desarrollo. Recuperado de: http://www.un.org/popin/icpd/conference/offspa/sconf13.html

República de Colombia. Min Protección. Fondo de Población de las Naciones Unidas UNPFA. Servicios de Salud Amigables para Adolescentes y Jóvenes. (2008). Un modelo para adecuar las respuestas de los servicios de salud a las necesidades de adolescentes y jóvenes de Colombia. 2da. Edición. Recuperado de:

http://unfpa.org.co/wp-content/uploads/2013/09/modelosamigables2.pdf 
Ruiz, A., Muller, E. Guevara. O. (2009). Clinical simulation and virtual learning. Complementary technologies for medical education. Revista Facultad Medicina, 57, pp. 67-79. Recuperado de:

http://www.scielo.org.co/pdf/rfmun/v57n1/v57n1a09.pdf

Schutt, J. y Maddaleno, M. (2003). Salud Sexual y Desarrollo de Adolescentes y Jóvenes en las Américas: Implicaciones en programas y políticas Organización Panamericana de la Salud 2003.. Washington. D.C. Recuperado de:

http://cidbimena.desastres.hn/docum/ops/pdf/SSRA.pdf

Secretaría de Relaciones Exteriores de México. Fondo de Desarrollo de las Naciones Unidas para la Mujer (UNIFEM). Programa de las Naciones Unidad para el Desarrollo (PNUD) (2004).. Manual: Convención sobre la eliminación de todas las formas de discriminación contra las mujeres y su protocolo facultativo CEDAW. 2 da. Edición. ISBN: 0912917-79-4, pp. 18-19. Recuperado de: http://www.undp.org.mx/IMG/pdf/segediconvcedaw-2.pdf 